\title{
The Relationship of Arginine Deprivation, Argininosuccinate Synthetase and Cell Death in Melanoma
}

\author{
Niramol Savaraj ${ }^{1}$, Chunjing Wu², Marcus Tien Kuo ${ }^{3}$, Min You $^{2}$, Medhi Wangpaichitr ${ }^{2}$, \\ Carlos Robles ${ }^{1}$, Seth Spector ${ }^{1}$ and Lynn Feun ${ }^{2}$ \\ ${ }^{1}$ VA Medical Center, Hematology-Oncology, Miami, Florida, U.S.A. \\ ${ }^{2}$ University of Miami, Hematology-Oncology, Miami, Florida, U.S.A. \\ ${ }^{3}$ M.D. Anderson Cancer Center, Molecular Pathology, Houston, Texas, U.S.A.
}

\begin{abstract}
It has been shown that melanoma cells do not express argininosuccinate synthetase (ASS) and therefore are unable to synthesize arginine from citrulline. Depleting arginine using pegylated arginine deiminase (ADI-PEG20) results in cell death in melanoma but not normal cells. This concept was translated into clinical trial and responses were seen. However, induction of ASS expression does occur which results in resistance to ADI-PEG20. We have used 4 melanoma cell lines to study factors which may govern ASS expression. Although these 4 melanoma cell lines do not express ASS protein or mRNA as detected by both immunoblot and northernblot analysis, ASS protein can be induced after these cells are grown in the presence of ADI-PEG20, but again repressed after replenishing arginine in the media. The levels of induction are different and one cell line could not be induced. Interestingly, a melanoma cell line with the highest level of induction could also be made resistant to ADI-PEG20. This resistant line possesses high levels of ASS mRNA and protein expression which cannot be repressed with arginine. Our study indicates that ASS expression in melanoma cells is complex and governed by biochemical parameters which are different among melanoma cells.
\end{abstract}

Keywords: Melanoma, arginine deiminase, argininosuccinate synthetase.

\section{Introduction}

Treatment of advanced malignant melanoma has not significantly improved in the past 20 years. Most chemotherapeutic agents have response rates of $15-20 \%$ or less (DeVita et al. 2001). Immunotherapeutic agents including interleukin-2, adoptive immunotherapy, interferon alpha and vaccine therapy may produce significant responses in a very small subset of patients, but their overall response rate is not better than chemotherapy (Brinckerhoff et al. 2000; Riker et al. 2007). Combinations of chemotherapy and immunotherapy also have not produced significant response rates in randomized trials (Keilholz and Gore, 2002; Punt et al. 2006; Sasse et al. 2007). Recently, blocking of Raf signaling has been explored for the treatment of melanoma (Alsina et al. 2003; Hoeflich et al. 2006; Scott et al. 2000; Sridhar et al. 2005), however, the first generation of Raf inhibitors such as Sorafenib has not shown significant antitumor activity in malignant melanoma. Thus, new effective agents are needed for the treatment of this disease. We and others have shown that melanoma cell lines do not express argininosuccinate synthetase (ASS) (Ensor et al. 2002; Feun and Savaraj, 2006; Scott et al. 2000; Shen et al. 2006; Wheatley, 2005; Wheatley and Campbell, 2002; Wheatley and Campbell, 2003) a key enzyme which converts citrulline to argininosuccinate. Argininosuccinate is then converted to arginine by argininosuccinate lyase. Thus, melanoma cells are particularly vulnerable to arginine depletion while normal cells are able to survive. In fact, exposure of melanoma cells to arginine deiminase(ADI), an enzyme which catalyzes the hydrolysis of arginine to citrulline results in cell death (Ensor et al. 2002; Shen and Shen, 2006; Sugimura et al. 1992; Takaku et al. 1995; Takaku et al. 1993). This laboratory finding has been developed into clinical trial, and antitumor activity has been documented in melanoma patients (Ascierto et al. 2005; Feun and Savaraj, 2006). However, not all patients responded to treatment and the duration of response varied. In this communication we explore the role of ASS gene expression in ADI response/resistance using 4 melanoma cell lines as models.

Correspondence: Nirmal Savaraj, VA Medical Center, Hematology-Oncology, 1201 NW 16th Street, Miami, Florida 33125. Tel: 305-575-3143; Email: nsavaraj@med.miami.edu

Please note that this article may not be used for commercial purposes. For further information please refer to the copyright statement at http://www.la-press.com/copyright.htm 


\section{Material and Methods}

\section{Cell lines}

Four human melanoma cell lines were used: A375, SK-MEL-2, A2058 and MEL-1220. The first three cell lines (A375, Sk-Mel-2 and A2058) were from ATCC, and MEL-1220 was established in our laboratory from a subcutaneous biopsy of a melanoma patient. This cell line produces melanin pigment and is positive for S-100. A telomerase immortalized normal foreskin fibroblast cell line (BJ-1) was purchased from Clonetech to be used as normal control. NSCLCS was derived from metastatic adenocarcinoma to the brain from primary lung cancer. This cell line grows in monlayer and was positive for keratin and epithelial membrane antigen. All cells were cultured in MEM with 10\% FCS and Penicillin/Streptomycin. Cells were checked for mycoplasma every month using MycoAlert Mycoplasma Detection Kit purchased from Cambrex.

\section{Reagent}

ADI-PEG20 was supplied by Polaris Inc. LArg-free Medium was purchased from Invitrogen. ASS antibody was purchased from BD Bioscience. ASS antibody was purchased from BD-Biosciences. PARP antibody was purchased from Cell Signaling and actin antibody was purchased from Sigma.

\section{Growth inhibitory effect}

$0.6 \times 10^{5}$ cells were seeded onto 24 -well plates and allowed $6 \mathrm{hr}$. for attachment. Various concentrations of ADI were added to each well. Each concentration was performed in duplicate. After $72 \mathrm{hr}$. exposure, cells were counted in the presence of $0.2 \%$ trypan blue. The growth inhibitory effect (ID50) was determined by plotting the number of viable cells as a percentage of control against the compound concentration.

\section{Detection of apoptosis}

Cells $\left(1 \times 10^{4}\right)$ were seeded onto Lab-Tek chamber slides (NALGE NUNC International) and then exposed to ADI-PEG20 at $0.1 \mathrm{ug} / \mathrm{ml}$ for $72 \mathrm{hrs}$. The apoptotic cells were detected by an in situ endlabeling assay using a kit from ONCOR. Briefly, cells were fixed in $4 \%$ neutral buffered formalin, treated with $2 \%$ hydrogen peroxide, and incubated with terminal deoxynucleotide transferase enzyme and digoxigenin-11-dUTP under a plastic coverslip for $1 \mathrm{hr}$. Anti-digoxigenin peroxidase was applied to the slide, followed by the chromogenic substrate diaminobenzidine and counterstained with hematoxylin. Cells that underwent apoptosis showed dark brown staining in the nuclei. We have also used poly ADP-Ribose Polymerase cleavage assay to detect apoptosis. During apoptosis ICE family such as caspase- 3 and caspase-7 cleave PARP to yield 85 and $25 \mathrm{kDa}$ fragments. Briefly, cells were treated with ADI-PEG20 for $72 \mathrm{hrs,} \mathrm{nuclear} \mathrm{protein} \mathrm{was}$ obtained and immunoblot was performed using PARP rabbit polyclonal antibody purchased from Cell Signaling.

\section{Analysis of arginine and citrulline by HPLC}

Arginine and citrulline analysis was performed by cation exchange Hewlett Packard 1100 Series HPLC with a post-column derivitization(11). This instrument utilizes a cation exchange column and a guard column. The temperature was set at $34^{\circ} \mathrm{C}$ and the reactor temperature was set at $39^{\circ} \mathrm{C}$. Amino acid standards arginine and citrulline were prepared in Li220 diluent (all mobile phase reagents were obtained as pre-mixed preparations from Pickering Laboratories). The mobile phase reagents were Pump A Li280, Pump B Li750, Pump C RG003. The initial conditions were $100 \%$ A for 12 min., followed by a linear gradient of 0 $15 \% \mathrm{~B}$ over the next $16 \mathrm{~min}$. The mobile phase was then switched to $92 \% \mathrm{~B}$ and $8 \% \mathrm{C}$ and an isocratic gradient was run for an additional $37 \mathrm{~min}$. A constant flow rate of $0.3 \mathrm{~mL} /$ minute was used.

\section{ASS expression detected by reverse transcriptase polymerase chain reaction}

Total RNA was extracted using the kit from Invitrogen. First-strand cDNA was generated from $0.2 \mathrm{ug}$ of total RNA using MLV Reverse-transcription. The amplification was carried out using the following primers: forward primer: GGCCAAAAAGGTGTTCATTG (nt: 240-259); reverse primer: ATTCCAATGAAGCGGTTCTC (nt: 883-902) and set as follows: denature: $45 \mathrm{sec}$. at $94^{\circ} \mathrm{C}$, annealing 45 second at $60{ }^{\circ} \mathrm{C}$ and extension $1 \mathrm{~min}$. at $72^{\circ} \mathrm{C}$ for a total of 30 cycles. GAPDH was used as control. The primers for GAPDH were as follows: 
forward primer: GAAGGTGAAGGTCGGAGTC; reverse primer: CAAAGTTGTCATGGATGACC.

\section{Southernblot analysis of ASS gene}

$10 \mathrm{ug}$ of nuclear DNA were digested with EcoRI restriction enzyme and electrophoresed using $0.8 \%$ agarose gels at $3 \mathrm{~cm} / \mathrm{V}$ for $10-16 \mathrm{hrs}$ in TAE buffer (40 mM Tris-acetate, $1 \mathrm{mM}$ EDTA, pH8.0). The nuclear DNA was transferred onto a nylon filter (Hybond-N; Amersham) and hybridized with ${ }^{32} \mathrm{P}-\mathrm{dCTP}$ ASS probe overnight, washed, and autoradiography was performed. ASS probe was generated from BJ-1 cell line. Briefly, the ASS PCR product was obtained using the primers mentioned above, then eluted from the agorose gel using the kit from Qiagen and labeled by ${ }^{32} \mathrm{P}-\mathrm{dCTP}$ using random oligolabeling technique.

\section{Northernblot analysis of ASS gene}

Total RNA was extracted using the kit from Invitrogen. $10 \mathrm{ug}$ total RNA was separated in 1\% agarose gel in MOPS -formaldehyde buffer, transferred to a nylon membrane (Hybond-N; Amersham) and hybridized with ${ }^{32} \mathrm{P}-\mathrm{dCTP}$ labeled ASS probe. Autoradiography was carried out.

\section{Westernblot analysis of ASS}

Cells were lysed with RIPA buffer (10 mMTris pH7.4 $100 \mathrm{mM} \mathrm{NaCl}, 1 \mathrm{mM}$ EDTA, $20 \mathrm{mM}$ $\mathrm{Na}_{4} \mathrm{P}_{2} \mathrm{O}_{7}, 2$ mM Na $3 \mathrm{VO}_{4}, 1 \%$ NP-40, 0.5\% deoxycholate, $1 \mathrm{mM}$ PMSF) and protease inhibitor cocktail from Sigma, passed several times through a $23 \mathrm{G}$ needle, and centrifuged. The total protein was separated on $12 \%$ SDS-PAGE, transferred onto membrane and immunoblot with a specific antibody and detected by chemiluminescence.

\section{Construction of human argininosucci- nate synthetase expression vector}

To construct pcDNA3-ASS, we amplified the human argininosuccinate synthetase mRNA sequence from 79 to 1314 nt (using GenBank accession number X01630 as the reference) by PCR with primer pairs containing sequences 5'GCGGCCGC TCCAGCAAAGGC TCCGTGG (sense; underscore sequence contains the Not I site) and 5'-GCGGCCGCTATTTGGCAGTGACCTTGC (antisense; underscore sequence contains Not I site) using human pancrease cDNA (Clonetech, Mountain View, CA) as the template. The PCR product was cloned into PCR®II-TOPO vector (Invitrogen). The NotI fragment containing the respective cDNA was then transferred into the Not I site of CIN-HA-pcDNA3 vector, which contains a hemagglutinin tag, enhancer CIN sequence for expression, and a neomycin resistance marker for transfection selection.

Transfection of ASS gene. ASS cDNA was introduced into A375 and A2058 cell line using lipofectamine from Invitrogen and selected with G-418. These transfectants were then tested for their sensitivity to ADI-PEG20.

\section{Results}

\section{Growth inhibitory effect of ADI-PEG20}

The growth inhibitory effect as well as the arginine and citrulline concentrations in the media are shown in Table I. The ID50 ranged from 0.05$0.08 \mathrm{ug} / \mathrm{ml}$. However, there are no viable cells in all three cell lines (A375, Sk-Mel-2 and Mel-1220) after exposure to ADI-PEG20 for seven days, whereas in A2058 there is $2-3 \%$ viable cell left. From the ID50 results, it appears that in Sk-Mel-2 and A2058 cell lines require more ADI-PEG20 to deplete arginine in the media. At $72 \mathrm{hr}$. there is still arginine remaining in the media (12.8 uM for SkMel-2 and 23.5 uM for A2058) whereas in A375 and MEL-1220 there is no detectable arginine level in the media. These findings may be related to the capability of the intracellular machinery to maintain

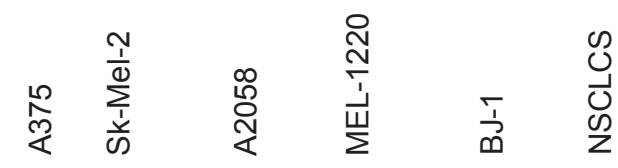

ASS

ACTIN

Figure 1. Immunoblot of ASS protein using commercially available monoclonal antibody (BD-Bioscience). All four melanoma cell lines (A375, Sk-Mel2, A2058 and MEL-1220) do not express ASS protein whereas NSCLCS and BJ-1 possess high levels of ASS protein. Actin was used as control. 
arginine either by degradation of certain unessential proteins or turning on ASS or other unknown mechanisms. All these 4 melanoma cell lines do not express ASS protein (as detected by westernblot, Fig.1) and neither at the transcriptional levels as detected by northernblot analysis. This data also correspond to what has been reported in the literature (Dillon et al. 2004; Ensor et al. 2002). However, the ASS DNA can be detected readily by southernblot analysis (Fig. 2). Thus, lack of ASS expression is not from gene deletion. In contrast, degradation of arginine by ADI-PEG20 did not have growth inhibitory effect on normal fibroblast BJ-1 and NSCLCS cell line (Table 1). These two cell lines possess high levels of ASS and hence are able to synthesize arginine from citrulline. These two cell lines also have arginine in the media at $0.05 \mathrm{ug} / \mathrm{ml}$ of ADI-PEG20. At this concentration there is no arginine in the media when EMEM media was incubated with ADI-PEG20 alone with no cells for 3 days.

\section{ADI induces apoptosis in melanoma cell line}

We have exposed melanoma cells to arginine free EMEM media with citrulline and $\mathrm{NH}_{4} \mathrm{Cl}$ supplement or ADI at $0.05 \mathrm{ug} / \mathrm{ml}$ for 3 days and assay for apoptosis. The results are shown in Figure 3A \& $3 \mathrm{~B}$. All melanoma cell lines undergo apoptosis by both PARP assay and in situ end labeling assay whereas BJ-1 and NSCLCS cells do not undergo apoptosis (data not shown).

\section{Arginine deprivation induces mela- noma ASS gene expression}

It has been shown that arginine levels can regulate ASS expression in lymphoblastoma cells

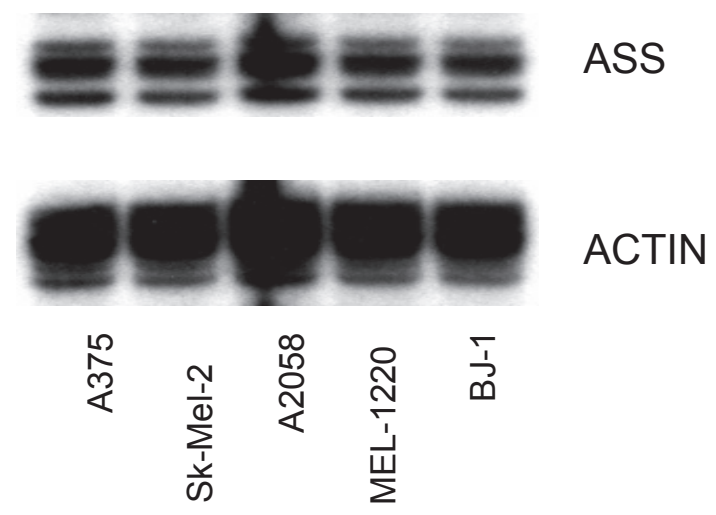

Figure 2. Southernblot Analysis of ASS in 4 melanoma cell lines and BJ-1 cell lines. All five cell lines show similar levels of ASS DNA.

and epithelial cells (Jackson et al. 1996; Philip et al. 2003). However, whether this phenomenon occurs in melanoma cells which lack ASS expression is not known. We have studied this possibility by exposing four melanoma cell lines (A375, Sk-Mel-2, A2058 and MEL-1220) to ADI-PEG20 for 3 days and assay for ASS expression. The results are shown in Figure 4. It is of interest that despite the similar levels of arginine deprivation, the levels of ASS expression as detected by westernblot are different. A2058 has more ASS expression (2.76 fold) followed by Sk-Mel-2 (2.03) and A375(1.52) while MEL-1220 is unable to turn on ASS gene. To further confirm this finding we have cultured all four cell lines in the arginine free media with or without citrulline $(0.4 \mathrm{uM})$ and $\mathrm{NH}_{4} \mathrm{Cl}(0.4 \mathrm{uM})$ for $72 \mathrm{hr}$. as well as using MEM media which has been incubated with 0.05 ug of ADI-PEG20 for $72 \mathrm{hr}$. to deplete arginine. At 24, 48 and $72 \mathrm{hr}$., cells were harvested and assayed for ASS expression. After $72 \mathrm{hrs}$ cells were placed on normal EMEM media. At 24, 48 and 72 hrs after being in normal media cells were harvested

Table I. Growth Inhibitory Effect of ADI-PEG20.

\begin{tabular}{llll}
\hline Cell lines & ID50(ug/ml) & $\begin{array}{l}\text { Arginine uM } \\
\mathbf{( 7 2} \mathbf{~ h})\end{array}$ & $\begin{array}{l}\text { Citrulline uM } \\
\mathbf{( 7 2} \mathbf{~ h})\end{array}$ \\
\hline A2058 & $0.088 \pm 0.008$ & 23.5 & 367.5 \\
A375 & $0.055 \pm 0.001$ & ND & 317.5 \\
Sk-Mel-2 & $0.07 \pm 0.008$ & 12.8 & 329.2 \\
MEL-1220 & $0.09 \pm 0.005$ & 0 & 3 \\
BJ-1 & $>1$ & 1.6 & 404 \\
NSCLCS* & $>1$ & 20.27 & 603 \\
\hline
\end{tabular}



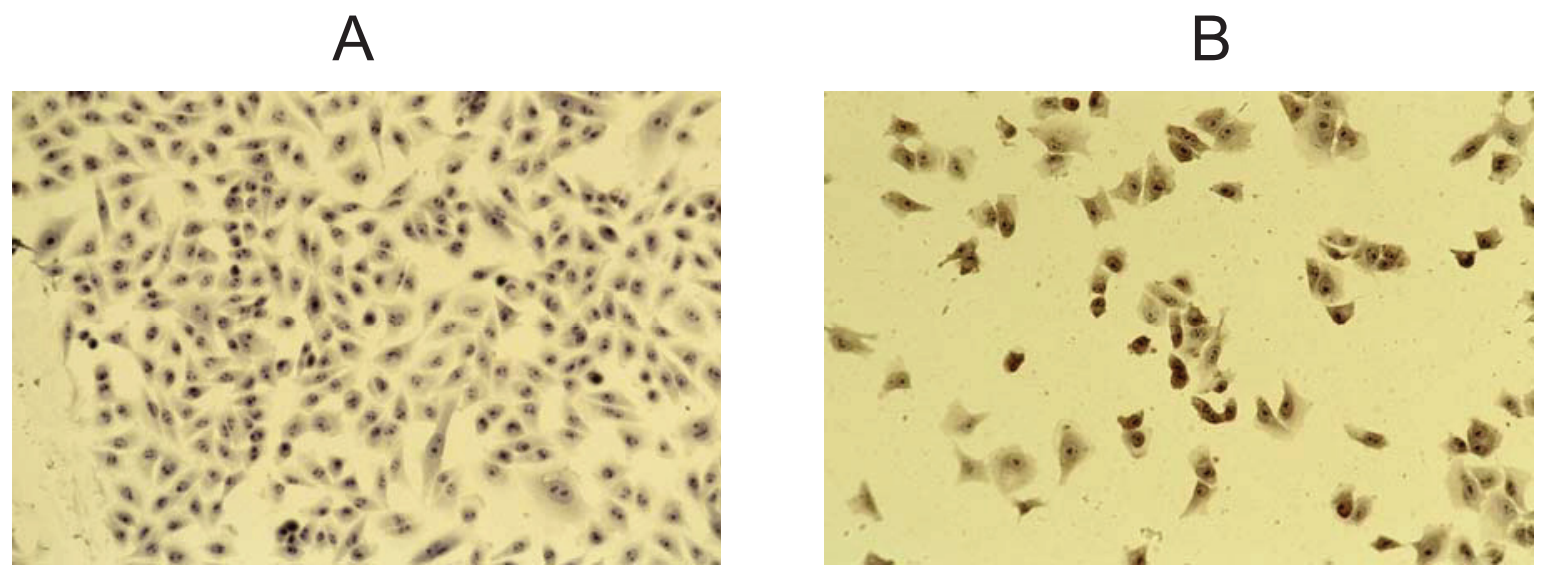

Figure 3A. In situ end labeling apoptosis assay in A375 cell line. A: control. B: after exposure to $0.08 \mathrm{ug} / \mathrm{ml}$ for $72 \mathrm{hr}$. Treated cells undergo apopotosis shown brown staining in the nuclei.

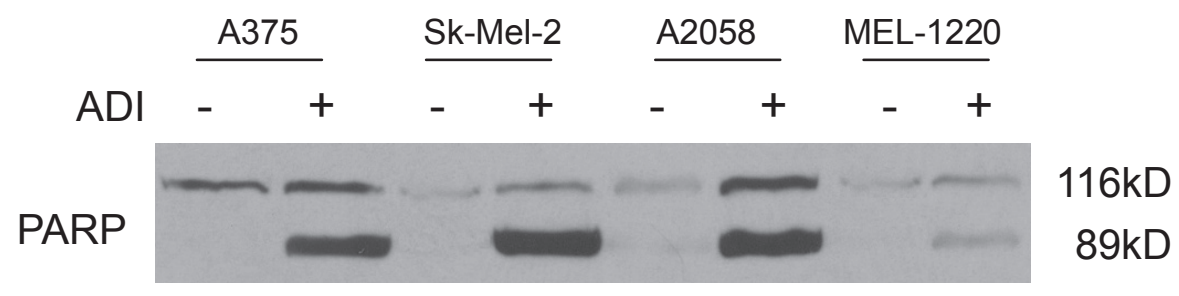

Actin

Figure 3B. Apoptosis as detected by PARP cleavage in 4 melanoma cell lines (A375, Sk-Mel-2, A2058 and MEL-1220). Untreated cell showed uncleaved PARP at $116 \mathrm{kD}$ whereas treated cells showed cleaved PARP seen at $89 \mathrm{kD}$.

and assayed for ASS expression. The results are shown in Figure 5. The three type of media yield similar results. Similar to data in Figure 4, A2058 appears to have highest ASS levels in arginine free media followed by Sk-Mel-2 and A375, while MEL1220 has no effect (data not shown). Thus, our results indicate that the levels of arginine in the media regulate ASS protein levels, and that the amount of citrulline did not have any effect. Interestingly, ASS gene expression cannot be detected by northernblot analysis (Fig. 8), but may be slightly increased by RT-PCR (data not shown). Thus, it appears that increased translation of ASS proceeds transcription.

\section{Arginine deprivation does not induce ASS expression in NSCLC}

We further investigated whether arginine deprivation also induced ASS expression in tumor cell lines which constitutively express ASS. We have chosen NSCLCS which was established from metastatic adenocarcinoma of the lung to the brain. This cell line expresses ASS and is not sensitive to ADI-PEG20. Cells were seeded in arginine free media with citrulline supplement or ADI-PEG20 treated media to degrade all arginine for 3 day and assay for ASS expression by westernblot. The results are shown in Figure 6. ASS expression is similar in normal media or arginine depleted media. Thus, it appears that arginine deprivation does not have effect on ASS expression in tumor cell lines which constitutively express high levels of ASS.

\section{ASS gene transfection results in resistance to ADI}

We have transfected ASS cDNA into A375, and A2058, and then assay for growth inhibitory effect of ADI-PEG20. We are able to obtain only 2 fold increase in ASS expression as shown by RT-PCR and westernblot (Fig. 7A \& 7B). The ID50 of these 


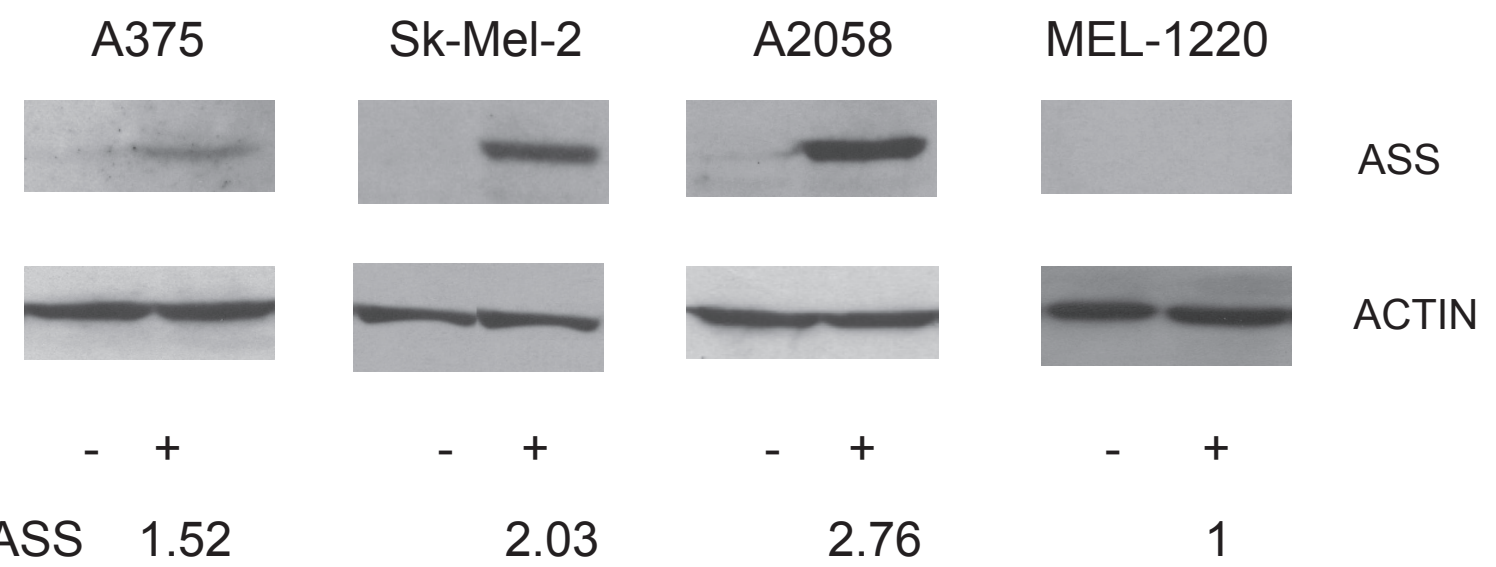

Ratio of ASS 1.52

2.03

2.76

Figure 4. Immunoblot of ASS protein in 4 melanoma cell lines (A375, Sk-Mel-2, A2058 and MEL-1220) before and after exposure to ADI-PEG20 for 3 days. The ratio of ASS depicts the intensity of ASS expression by densitometer after exposure to ADI versus control background. A2058 exhibited the highest level of expression whereas MEL-1220 did not express ASS after exposure to ADI-PEG20.

transfectants were $0.1 \pm 0.05 \mathrm{ug} / \mathrm{ml}$ for A375, and $0.2 \pm 0.05 \mathrm{ug} / \mathrm{ml}$ for A2058, respectively. Although we are unable to yield high levels of ASS expression in these transfectants, this modest increase in ASS expression does affect slightly the ID50 of ADI-PEG20 with 2 fold increase.

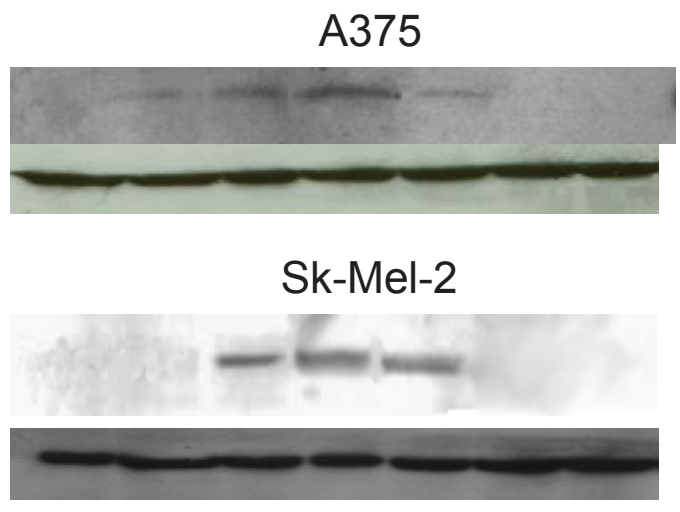

ASS ACTIN

\section{A2058}

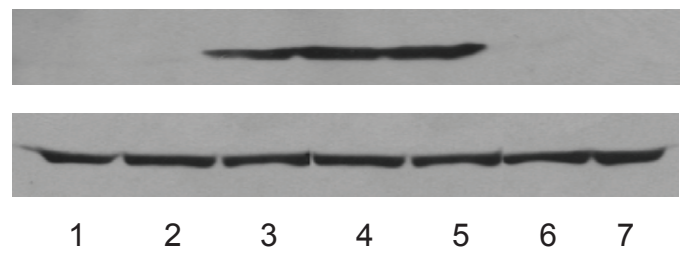

ASS

ACTIN

Figure 5. Immunoblot of ASS protein in 3 melanoma cell lines (A375, Sk-Mel-2, A2058) before and after exposure arginine free media supplemented with citrulline and $\mathrm{NH}_{4} \mathrm{Cl}$ for 24,48 and $72 \mathrm{hrs}$. Afterward, cells were washed, and replenished with normal EMEM media for 24, 48 and $72 \mathrm{hrs}$. Lane 1: control. Lane 2: $24 \mathrm{hrs}$ on arginine free media Lane 3: 48hrs on arginine free media Lane 4: $72 \mathrm{hrs}$ in arginine free media Lane 5: Removal of arginine free media and changed to normal EMEM media for $24 \mathrm{hrs}$. Lane 6: $48 \mathrm{hrs}$ in normal media. Lane 7: $72 \mathrm{hrs}$ in normal media. Similar results were obtained with arginine free media with no citrulline supplement and ADI-PEG20 treated media.

\section{Generation of ADI-PEG20 resistant cell line}

Since we were unable to generate a stable ASS transfected cell line with high levels of ASS expression, we have exposed A2058 cell lines to ADI-PEG20 at $0.05 \mathrm{ug} / \mathrm{ml}$ for 5 days with 2 days off $\times 6$ weeks. We were able to establish A2058R with high levels of ASS expression by northernblot (Fig. 8A) and by westernblot analysis (Fig. 8B). This cell line is resistant to ADI-PEG20 with ID50 $>1 \mathrm{ug} / \mathrm{ml}$. Interestingly, the levels of ASS protein also are not affected by arginine deprivation (data not shown).

\section{Discussion}

It has been reported that melanoma cell lines as well as tumor samples do not express ASS and thus are auxotrophic for arginine. Arginine deprivation using arginine deiminase or arginase has

\section{NSCLCS}

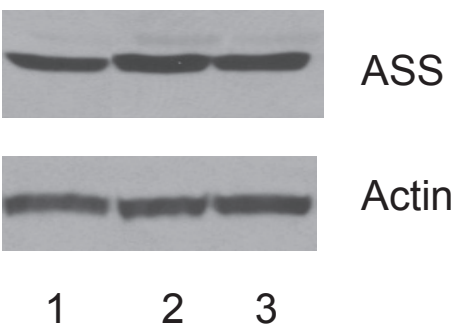

Figure 6. Immunoblot of ASS in NSCLCS: lane 1: control (normal media ). lane 2: ADI-PEG20 treated media for $72 \mathrm{hr}$. Lane 3 : After exposure to arginine free media with citrulline and $\mathrm{NH}_{4} \mathrm{Cl}$ supplement for $72 \mathrm{hrs}$. 


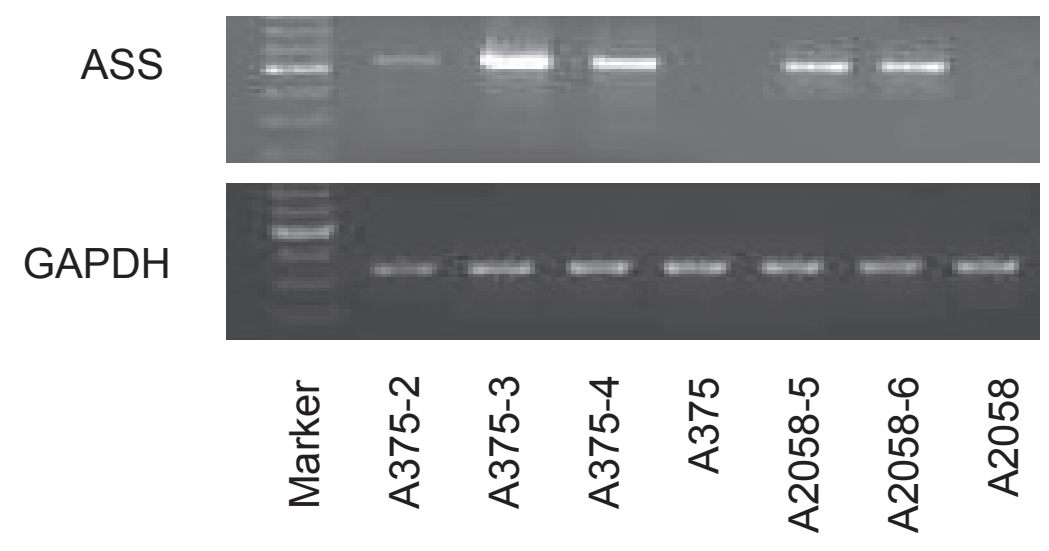

Figure 7A. RT-PCR of ASS in A375 and A2058 and their ASS transfected clones A375-2, A375-3, A375-4. and A2058-5,A2058-6, respectively. A375-3, and A2058-6 possess high levels of expression and were selected to study the growth inhibitory effect of ADI-PEG20.

been shown by several investigators to have antitumor activity in melanoma cell lines as well as in other tumor cell lines which lack ASS in vitro (Dillon et al. 2004; Ensor et al. 2002; Gong et al. 2000; Miyazaki et al. 1990; Noh et al. 2004; Shen et al. 2006; Sugimura et al. 1992; Szlosarek et al. 2006; Takaku et al. 1995; Takaku et al. 1993; Yoon et al. 2007). Correlation of antiproliferative effect of ADI-PEG20 with endogenous ASS levels also has been reported (Ensor et al. 2002; Shen et al. 2003). However, these enzymes have a short half life and high antigenicity which makes them not suitable for in vivo use. To overcome this problem, a pegylated form of ADI (ADI-PEG20) was developed by Polaris Inc. and has been shown to increase the half life as well as decrease the antigenicity (Izzo et al. 2004). This compound has been shown to have antitumor activity in vivo (Ensor et al. 2002). Clinical trials also look promising with antitumor responses seen in a number of melanoma patients (Ascierto et al. 2005; Feun and Savaraj, 2006). However, not all patients respond to ADI-PEG20 and resistance to this compound does occur after treatment. Our limited clinical data suggest that ASS expression occurs in patients who develop resistance to ADI-PEG20 (Feun and Savaraj, 2006). Thus, it appears that exposure to ADI-PEG20 which results in continuous arginine depletion in the serum results in ASS expression and hence drug resistance. In this communication, we have shown that exposure of melanoma cells to ADI-PEG20 or arginine free media can result in ASS protein production which again become negligible when cells were exposed to normal media. Interestingly, the levels of ASS protein being induced while cells are deprived with arginine vary among the 4 melanoma cell lines. A2058 has the highest of ASS protein followed by Sk-Mel-2 and A375. One melanoma cell line (MEL -1220) cannot produce ASS protein despite being cultured in the arginine depleted media. Interestingly, A2058 can be selected to become resistant to ADI-PEG20 rather rapidly in 6 weeks. The primary mechanism of resistance involves increase in ASS protein production followed by ASS transcription as detected by northernblot analysis. The resistance also is irreversible despite replacement with normal EMEM media. On the other hand, MEL-1220 cannot be made resistant to ADIPEG20. These findings may mirror what we have found in melanoma patients. This is the first report which demonstrated that extracellular arginine does control ASS protein in certain melanoma cells. The underlying mechanism(s) on how arginine controls ASS translation/transcription in melanoma are not known. It is possible that when the arginine levels is low, the cells will attempt to increase the translation of ASS in order to synthesize arginine from citrulline. We are

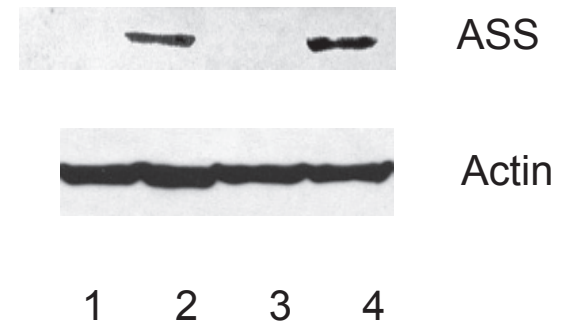

Figure 7B. Immunoblot of ASS in control and transfected cells. Lane 1: A-375. Lane 2: A375-3. Lane 3: A2058. Lane 4: A2058-6 (A375-3 and A2058-6 transfected with ASS). 


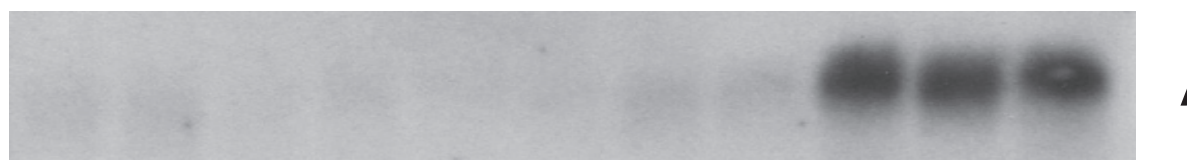

ASS
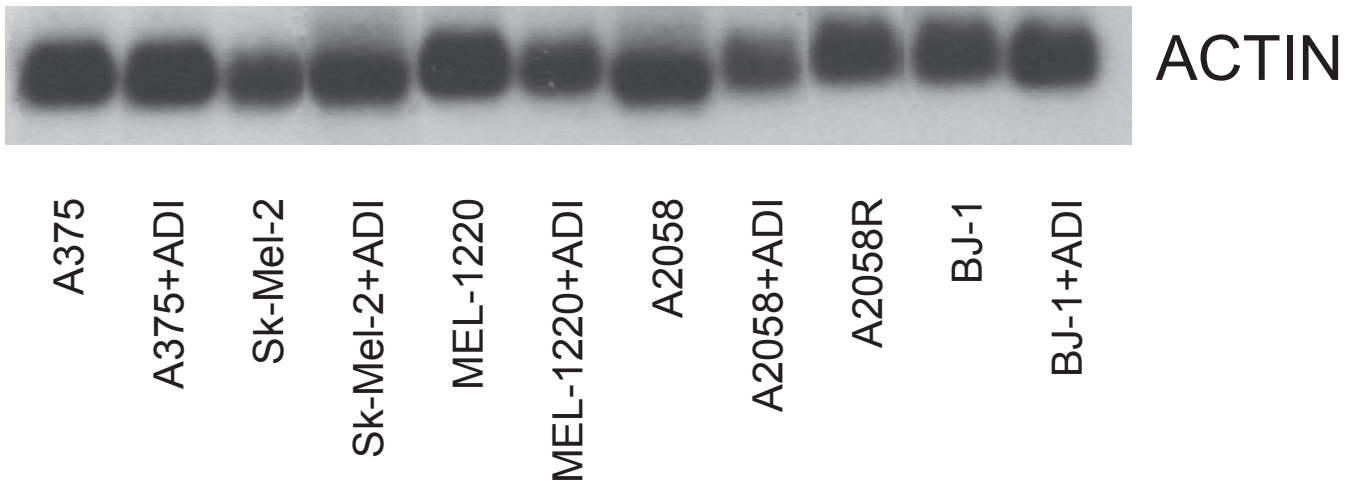

Figure 8A. Northernblot Analysis of ASS in a panel of melanoma cell line and BJ-1 cells. Lane 1: A375. Lane 2: A375 after exposure to ADI-PEG20 for 72hrs. Lane 3: SK-Mel-2 Lane 4: Sk-Mel-2 after exposure to ADI-PEG20 for 72 hrs. Lane 5: MEL-1220. Lane 6: MEL-1220 after exposure to ADI-PEG20 for 72 hrs. Lane 7: A2058. Lane 8: A2058 after exposure to ADI-PEG20 for 72 hrs. Lane 9: A2058R. Lane 10: BJ-1. Lane 11: BJ-1 after exposure to ADI-PEG20 for $72 \mathrm{hrs}$. Note: only A2058R and BJ-1 possess $1.9 \mathrm{~kb}$ ASS mRNA and there is no differences in ASS mRNA in BJ-1 cells after exposure to ADI-PEG20.

currently investigating both transcription and translation control of ASS in both normal and arginine free media. Interestingly, the levels of induction are different among the melanoma cell lines tested. It appears that the cell line which has high levels of induction is capable of becoming resistant to ADI-EG20 in a short period of time (within 6 weeks). On the other hand, the cell line which has no induction of ASS (MEL-1220) is not capable of turning on ASS gene transcription and becoming resistant to ADI-PEG20. Whether these findings occur in patients' tumor samples is not known. However, we have observed a melanoma patient who was ASS(-) prior to treatment and become ASS positive at the time of treatment failure which suggest that arginine deprivation

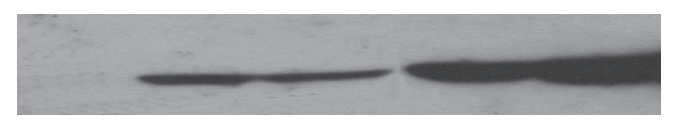

ASS

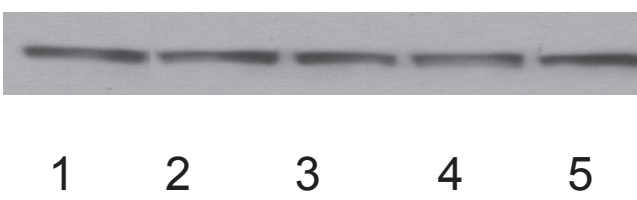

ACTIN

Figure 8B. Immunoblot of ASS. Lane 1: A2058. Lane 2: A2058 in arginine free media with out citrulline and $\mathrm{NH}_{4} \mathrm{Cl}$ 2. Lane 3: $\mathrm{A} 2058$ in arginine free media supplemented with citrulline and $\mathrm{NH}_{4} \mathrm{Cl}$. Lane 4: A2058R. Lane 5: BJ-1 cells. ASS protein in A2058R is only slightly less than BJ-1. can result in ASS expression. As mention earlier, the mechanism by which arginine in the media control ASS expression is not known, we are currently investigating how ASS gene transcription is regulated in the presence and absence of arginine.

ASS cDNA has been cloned in 1981 (Su et al. 1981). The kinetic properties of ASS enzyme has been extensively studied and the crystal structure in bacteria has been identified (Husson et al. 2003; Ratner, 1973). The transcriptional and translational control of ASS gene are not well understood and appear to be tissue specific (Husson et al. 2003). Several hormones such as glucocorticoids, glucagons, insulin and fatty acid have been shown to positively or negatively influence ASS expression (Husson et al. 1975; Husson et al. 1986; Husson and Vaillant, 1979; Husson and Vaillant, 1982; Lin et al. 1982). Our preliminary data does not indicate that these hormones influence ASS expression in melanoma cells. However, arginine in the media can mediate ASS expression in certain melanoma cells. In contrast, arginine in the media has no effect in A2058R (selected by ADI-PEG20) or NSCLCS, both have high levels of ASS expression. This finding is similar to the previous report (Boyce et al. 1986) which showed that arginine mediated ASS repression does not occur in canavanine (arginine analog) resistant variant of RPMI 2650 cells which has high levels of ASS expression. 
Nevertheless, the key question remains what are the factors which govern ASS expression in melanoma cells. If these factors can be identified, one can attempt to repress the ASS expression and hence evade ADI-PEG20 resistance.

It is not yet clear why transfection with ASS cDNA in melanoma cells did not yield high levels of ASS expression and we are unable to generate stable transfected cell line(s). It is conceivable that certain factors in melanoma cells prevent this cDNA to express at high levels and/or arginine in the media may also play a role in repressing the expression of transfected ASS. We are currently investigating these possibilities.

\section{Acknowledgment}

This work was supported by a grant (R01CA 109578) from NIH and a research grant from VA. We would like to thank Polaris Inc. for providing ADI-PEG20 and analysis of arginine and citrulline.

\section{References}

Alsina, J., Gorsk, D.H., Germino, F.J. et al. 2003. Detection of mutations in the mitogen-activated protein kinase pathway in human melanoma. Clin. Cancer Res., 9:6419-25.

Ascierto, P.A., Scala, S., Castello, G. et al. 2005. Pegylated arginine deiminase treatment of patients with metastatic melanoma: results from phase I and II studies. J. Clin. Oncol., 23:7660-8.

Boyce, F.M., Anderson, G.M., Rusk, C.D. et al. 1986. Human argininosuccinate synthetase minigenes are subject to arginine-mediated repression but not to trans induction. Mol. Cell. Biol., 6:1244-52.

Brinckerhoff, L.H., Thompson, L.W. and Slingluff, C.L. Jr. 2000. Melanoma vaccines. Curr. Opin. Oncol., 12:163-73.

DeVita, V.T., Hellman, S. and Rosenberg, S.A. 2001. Cancer, principles and practice of oncology. Philadelphia: LIppincott, Williams \& Wilkins.

Dillon, B.J., Prieto, V.G., Curley, S.A. et al. 2004. Incidence and distribution of argininosuccinate synthetase deficiency in human cancers: a method for identifying cancers sensitive to arginine deprivation. Cancer, 100:826-33

Ensor, C.M., Holtsberg, F.W., Bomalaski, J.S. et al. 2002. Pegylated arginine deiminase (ADISS PEG20,000 mw) inhibits human melanomas and hepatocellular carcinomas in vitro and in vivo. Cancer Res., 62:5443-50

Feun, L. and Savaraj, N. 2006. Pegylated arginine deiminase: a novel anticancer enzyme agent. Expert. Opin. Investig. Drugs, $15: 815-22$

Gong, H., Zolzer, F., von, Recklinghausen, G. et al. 2000. Arginine deiminase inhibits proliferation of human leukemia cells more potently than asparaginase by inducing cell cycle arrest and apoptosis. Leukemia, 14:826-9.

Hoeflich, K.P., Gray, D.C., Eby, M.T. et al. 2006. Oncogenic BRAF is required for tumor growth and maintenance in melanoma models. Cancer Res., 66:999-1006.

Husson, A., Gautier, C. and Vaillant R. 1975. [Control activities and regulation by the glucocorticoids of three urea-cycle enzymes in rat foetal liver: argininosuccinate synthetase, argininosuccinase and arginase (author's transl)]. Experientia, 31:1403-5.

Husson, A. and Vaillant, R. 1979. Hormonal regulation of three urea cycle enzymes in rat fetal liver. Biol. Neonate., 35:74-81.
Husson, A. and Vaillant, R. 1982. Effects of glucocorticosteroids and glucagon on argininosuccinate synthetase, argninosuccinase, and arginase in fetal rat liver. Endocrinology, 110:227-32.

Husson, A., Guechairi, M., Fairand, A. et al. 1986. Effects of pancreatic hormones and glucocorticosteroids on argininosuccinate synthetase and argininosuccinase activities of rat liver during the perinatal period: in vivo and in vitro studies. Endocrinology, 119:1171-7.

Husson, A., Brasse-Lagnel, C., Fairand, A. et al. 2003. Argininosuccinate synthetase from the urea cycle to the citrulline-NO cycle. Eur. J. Biochem., 270:1887-99.

Izzo, F., Marra, P., Beneduce, G. et al. 2004. Pegylated arginine deiminase treatment of patients with unresectable hepatocellular carcinoma: results from phase I/II studies. J. Clin. Oncol., 22:1815-22.

Jackson, M.J., Zielke, H.R. and Zielke, C.L. 1996. Induction of astrocyte argininosuccinate synthetase and argininosuccinate lyase by dibutyryl cyclic AMP and dexamethasone. Neurochem. Res., 21:1161-5.

Keilholz, U. and Gore, M.E. 2002. Biochemotherapy for advanced melanoma. Semin. Oncol., 29:456-61.

Lin, R.C., Snodgrass, P.J. and Rabier, D. 1982. Induction of urea cycle enzymes by glucagon and dexamethasone in monolayer cultures of adult rat hepatocytes. J. Biol. Chem., 257:5061-7.

Miyazaki, K., Takaku, H., Umeda, M. et al. 1990. Potent growth inhibition of human tumor cells in culture by arginine deiminase purified from a culture medium of a Mycoplasma-infected cell line. Cancer Res., 50:4522-7.

Noh, E.J., Kang, S.W., Shin, Y.J. et al. 2004. Arginine deiminase enhances dexamethasone-induced cytotoxicity in human T-lymphoblastic leukemia CCRF-CEM cells. Int. J. Cancer, 112:502-8.

Philip, R., Campbell, E. and Wheatley, D.N. 2003. Arginine deprivation, growth inhibition and tumour cell death: 2. Enzymatic degradation of arginine in normal and malignant cell cultures. Br. J. Cancer, 88:613-23.

Punt, C.J., Suciu, S., Gore, M.A. et al. 2006. Chemoimmunotherapy with dacarbazine, cisplatin, interferon-alpha2b and interleukin-2 versus two cycles of dacarbazine followed by chemoimmunotherapy in patients with metastatic melanoma: a randomised phase II study of the European Organization for Research and Treatment of Cancer Melanoma Group. Eur. J. Cancer, 42:2991-5.

Ratner, S. 1973. Enzymes of arginine and urea synthesis. Adv Enzymol Relat Areas Mol. Biol., 39:1-90.

Riker, A.I., Radfar, S., Liu, S. et al. 2007. Immunotherapy of melanoma: a critical review of current concepts and future strategies. Expert. Opin. Biol. Ther, 7:345-58.

Sasse, A., Sasse, E., Clark, L. et al. 2007. Chemoimmunotherapy versus chemotherapy for metastatic malignant melanoma. Cochrane Database Syst. Rev., CD005413.

Scott, L., Lamb, J., Smith, S. et al. 2000. Single amino acid (arginine) deprivation: rapid and selective death of cultured transformed and malignant cells. Br. J. Cancer, 83:800-10.

Shen, L.J., Lin, W.C., Beloussow, K. et al. 2003. Resistance to the antiproliferative activity of recombinant arginine deiminase in cell culture correlates with the endogenous enzyme, argininosuccinate synthetase. Cancer Lett., 191:165-70.

Shen, L.J., Beloussow, K. and Shen, W.C. 2006. Modulation of arginine metabolic pathways as the potential anti-tumor mechanism of recombinant arginine deiminase. Cancer Lett., 231:30-5.

Shen, L.J. and Shen, W.C. 2006. Drug evaluation: ADI-PEG-20 - a PEGylated arginine deiminase for arginine-auxotrophic cancers. Curr Opin Mol Ther, 8:240-8 Sridhar SS, Hedley D and Siu LL. 2005. Raf kinase as a target for anticancer therapeutics. Mol. Cancer Ther., 4:677-85.

Su, T.S., Bock, H.G., O’Brien, W.E. et al. 1981. Cloning of cDNA for argininosuccinate synthetase mRNA and study of enzyme over production in a human cell line. J. Biol. Chem., 256:11826-31.

Sugimura, K., Ohno, T., Kusuyama, T. et al. 1992. High sensitivity of human melanoma cell lines to the growth inhibitory activity of mycoplasmal arginine deiminase in vitro. Melanoma. Res., 2:191-6

Szlosarek, P.W., Klabatsa, A., Pallaska, A. et al. 2006. In vivo loss of expression of argininosuccinate synthetase in malignant pleural mesothelioma is a biomarker for susceptibility to arginine depletion. Clin. Cancer Res., 12:7126-31. 
Takaku, H., Misawa, S., Hayashi, H. et al. 1993. Chemical modification by polyethylene glycol of the anti-tumor enzyme arginine deiminase from Mycoplasma arginini. Jpn. J. Cancer Res., 84:1195-200.

Takaku, H., Matsumoto, M., Misawa, S. et al. 1995. Anti-tumor activity of arginine deiminase from Mycoplasma argini and its growth-inhibitory mechanism. Jpn. J. Cancer Res., 86:840-6.

Wheatley, D.N. and Campbell, E. 2002. Arginine catabolism, liver extracts and cancer. Pathol. Oncol. Res., 8:18-25.

Wheatley, D.N. and Campbell, E. 2003. Arginine deprivation, growth inhibition and tumour cell death: 3 . Deficient utilization of citrulline by malignant cells. Br. J. Cancer, 89:573-6.

Wheatley, D.N. 2005. Arginine deprivation and metabolomics: important aspects of intermediary metabolism in relation to the differential sensitivity of normal and tumour cells. Semin. Cancer Biol., 15:247-53.

Yoon, C.Y., Shim, Y.J., Kim, E.H. et al. 2007. Renal cell carcinoma does not express argininosuccinate synthetase and is highly sensitive to arginine deprivation via arginine deiminase. Int. J. Cancer, 120:897-905. 